\title{
Is stroke etiology a predictor of successful recanalization status in acute endovascular therapy?
}

Karen D. Orjuela, MD¹. Wafik G. Sedhom². Gary Walker, MD¹. Patrick Hosokawa, MS³. Christopher Roark, MD³. ${ }^{1}$ Department of Neurology, ${ }^{2}$ School of Medicine, ${ }^{3}$ Department of Neurosurgery. University of Colorado Denver, Aurora, CO

\section{BACKGROUND:}

Endovascular treatment (EVT) using stentretriever therapies is considered the goldstandard approach for the treatment of acute ischemic stroke (AIS) in selected patient populations. The Thrombolysis in Cerebral Infarction ( $\mathrm{TICl}$ ) scale measures recanalization. Prior studies have demonstrated that a good neurological outcome depends on procedural success (considered a TICI2b or TICI3 score), collateral status and patient-specific factors. It remains unclear if stroke etiology is a predictor of procedural success ${ }^{1,2}$. We sought to determine if stroke etiology using the Trial of Org 10172 in Acute Stroke Treatment (TOAST) classification was an independent predictor of successful recanalization in acute EVT.

\section{METHODS:}

We reviewed our retrospectively collected endovascular database at the University of Colorado, Anschutz Medical Center for patients with acute ischemic strokes from January 2014 to June 2017 that underwent EVT. We evaluated baseline characteristics, procedural data, and TOAST classification to determine if there was an association between stroke etiology and $\mathrm{TICl}$ grade.

\begin{tabular}{|c|c|c|c|c|c|c|c|}
\hline $\begin{array}{c}\text { TOAST/ } \\
\text { TICI score }\end{array}$ & 0 & 1 & 2 & $2 \mathrm{a}$ & $2 \mathrm{~b}$ & 3 & $\begin{array}{c}\text { Mean TICI } \\
\text { score } \\
\text { (2a=2.2. } \\
2 \mathrm{~b}=2 . \mathrm{b})\end{array}$ \\
\hline 1 & & & & $6(21.4 \%)$ & $12(42.9 \%)$ & $10(18.2 \%)$ & 2.7 \\
\hline 2 & $4(80.0 \%)$ & $2(100.0 \%)$ & $1(50.0 \%)$ & $1(8.3 \%)$ & $12(25.5 \%)$ & $24(43.6 \%)$ & 2.5 \\
\hline 4 & & & & $2(16.7 \%)$ & $4(8.5 \%)$ & $7(12.7 \%)$ & 2.8 \\
\hline 5 & & & $1(3.9 \%)$ & $1(3.9 \%)$ & $14(29.8 \%)$ & $10(18.2 \%)$ & 2.7 \\
\hline $5 a$ & & & & $1(33.3 \%)$ & $1(33.5 \%)$ & $1(33.3 \%)$ & 2.6 \\
\hline $5 \mathrm{c}$ & $1(20.0 \%)$ & & & $1(8.3 \%)$ & $4(8.5 \%)$ & $3(5.5 \%)$ & 2.4 \\
\hline
\end{tabular}

Table 2: TOAST Vs TICI score
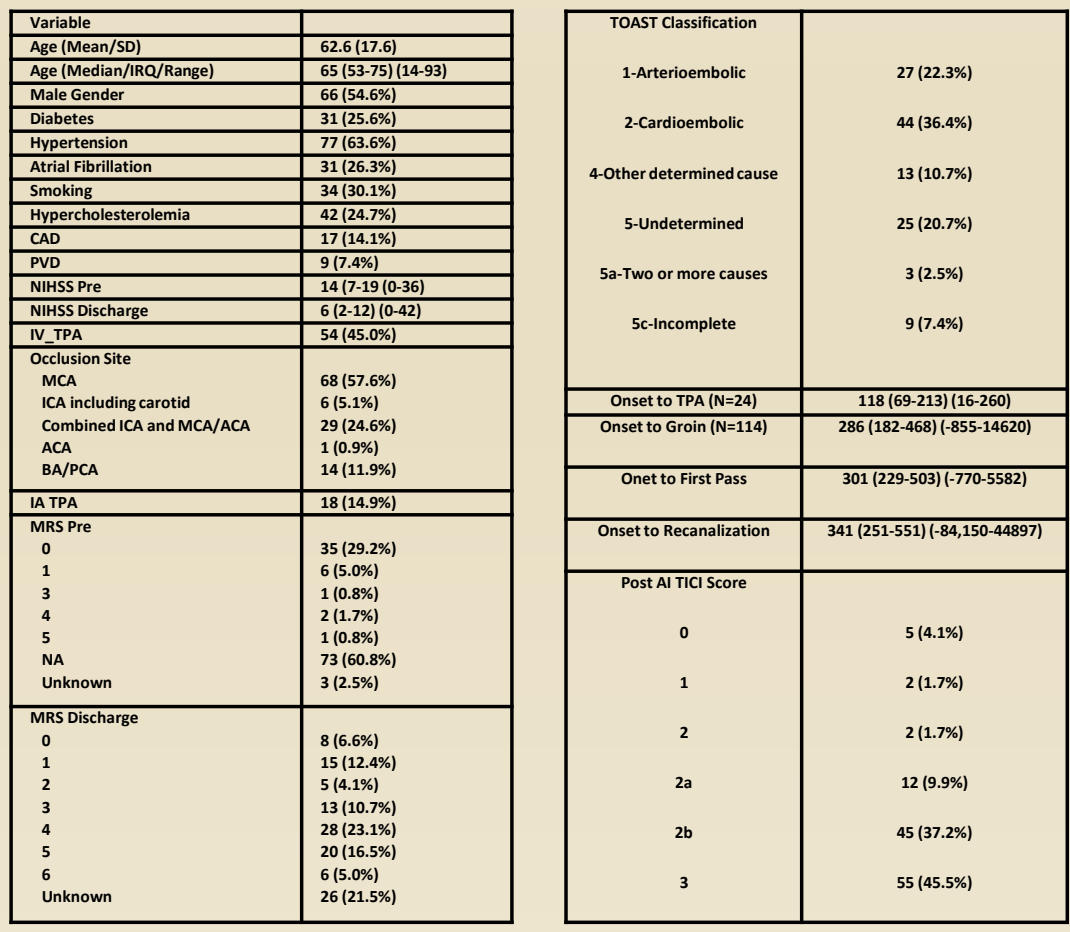

Table 1: Overall Summary ( $\mathrm{N}=\mathbf{1 2 1}$ )

A total of 121 patients were analyzed. Our analysis controlled for age, cardiovascular risk factors, previous antithrombotic therapy, baseline National Institutes of Health Stroke Scale score, intravenous thrombolytic therapy (IV tPA), time of onset to IV tPA, and time of onset to reperfusion. There was no association between stroke etiology determined by TOAST classification and successful recanalization status after statistical evaluation using ANOVA test $(F(5,117)=0.48$, $p=0.79$ ) and Chi Square: 0.34

\section{CONCLUSIONS:}

We did not find an association between stroke etiology and successful recanalization in this retrospective case series of over $\mathbf{1 2 0}$ consecutive EVT patients. 